\title{
Mendelian multiple sclerosis triggers mechanistic rethink
}

Evidence for rare Mendelian forms of multiple sclerosis (MS) has come from a new study published in PLOS Genetics. The mutations that were identified provide new insight into the underlying causes of MS and suggest a need for new therapeutic approaches.

Although MS aggregates in some families, the idea that single mutations can cause heritable MS has been controversial. In 2016, Carles Vilariño-Güell and colleagues identified a pathogenic mutation in one family with MS, suggesting that Mendelian forms of MS can exist. Now, Vilariño-Güell and his team have applied the same approach to a much larger group of families.

"Our aim was to determine the existence of Mendelian forms of MS and to identify genes and mutations causing disease that could provide a better understanding of the biological mechanisms that underlie MS onset," says Vilariño-Güell.

The researchers used whole- we may be targeting the with current treatments

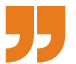
'wrong' system exome sequencing to analyse the genomes of 132 individuals with MS from a total of 34 different families. Following the identification of mutations, a further 2,502 people with MS were screened for the same mutations, and a database of MS-associated variants was screened for other mutations in the same genes.

In total, the analysis identified 12 genes linked with familial MS.

"These findings show that Mendelian forms of MS indeed exist," states Vilariño-Güell. "The mutations are extremely rare and have a reduced penetrance, but most of the people who had one of the mutations developed MS in their lifetime."

The 12 genes that were identified provide intriguing insights into the pathogenesis of MS. The genes relate to the fibrinolysis and complement pathways, inflammasome assembly, Wnt signalling, nuclear receptor complexes, and cation channels and exchangers, all of which contribute to interconnected immunological pathways.

"These mutations indicate that the root of the disease is not the adaptive immune response but the innate immune system," says VilariñoGüell. "Thus, we may be targeting the 'wrong' system with current treatments, as those address the symptoms but not the causes."

Identification of potentially causal mutations in MS provides the opportunity to create new animal models, and Vilariño-Güell says that this is the key next step. "Models based on human mutations have a greater potential to mimic the biological processes of disease in humans, and provide a better translational efficacy for new treatments," he concludes.

Ian Fyfe

ORIGINAL ARTICLE Vilariño-Güell, C. et al. Exome sequencing in multiple sclerosis families identifies 12 candidate genes and nominates biological pathways for the genesis of disease. PLOS Genet. 15, e1008180 (2019)

\section{NEURODEGENERATIVE DISEASE}

\section{Genetic variant cuts risk of multiple forms of dementia}

A rare genetic variant that protects against Alzheimer disease (AD) is also associated with reduced risks of other dementias, according to a new study published in Acta Neuropathologica. The finding could lead to the development of preventive treatments.

A specific variant of PLCG2, which is the gene that encodes phospholipase C $\gamma 2$ (PLC $\gamma 2$ ), has previously been associated with a reduced risk of AD. The PLC $\gamma 2$ protein is involved in the transduction of immune signals across cell membranes and is highly expressed in microglia. Given that the immune response is thought to have a role in multiple neurodegenerative diseases, the new study was designed to test whether the PLCG2 variant protects against other diseases as well as AD.

"The variant in PLCG2 is rare - it occurs in about $1 \%$ of the Dutch population," notes Sven van der Lee, who led the new study alongside Henne Holstege. "We reached out to collaborators worldwide and requested the genotype of the PLCG2 variant in patients with neurodegenerative diseases and controls." Using this approach, the team were able to analyse data from 53,000 patients, each of whom had one of seven different neurodegenerative diseases, and almost 150,000 healthy controls.

The presence of the PLCG2 variant was associated not only with a reduced risk of $A D$ but also with a reduced risk of dementia with Lewy bodies and frontotemporal dementia. The team did not detect an effect of the variant on the risk of progressive supranuclear palsy, amyotrophic lateral sclerosis, Parkinson disease or multiple sclerosis.

The variant was also associated with an increased chance of reaching at least 90 years of age and specifically with becoming a cognitively healthy centenarian, in whom the absence of dementia and extreme longevity is combined.

More work is required to understand the mechanism underlying the protective effect of the PLCG2 variant. "We suspect that the genetic variant leads to a change in the PLC $\gamma 2$ protein structure, which likely alters the functioning of PLC $\gamma 2$ in immune response processes," explains Holstege. "Now we need to find out how this variant keeps our brains healthy by investigating the effect of the PLCG2 variant in the relevant immune cells. Ultimately, we hope that mimicking the effect of the genetic variant might represent a first lead to agents that could decrease disease risk."

Sarah Lemprière to find out how this variant keeps our brains healthy A nonsynonymous mutation in PLCG2 reduces the risk of Alzheimer's disease, dementia with Lewy bodies and frontotemporal dementia, and increases the likelihood of longevity. Acta Neuropathol. https://doi.org/10.1007/s00401019-02026-8 (2019) 